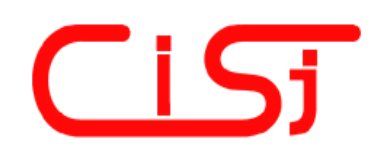

\title{
THE CHOICE AND SUBSTANTIATION OF THE MATHEMATICAL MODEL OF ELECTRORETINOGRAM IN THE FORM OF LINEAR STOCHASTIC PROCESS
}

\author{
S. V. Dzubin, A. V. Matsiuk, S. V. Martsenko, M. V. Pryimak \\ Ternopil State Technical University, Ruska str., 56, Ternopil, Ukraine, 46000 \\ kuba_molva@mail.ru,kaf_kn@tu.edu.te.ua
}

\begin{abstract}
The mathematical model of electroretinogram as a linear random process which takes into account the mechanism of eye retina biopotentials forming and is suitable for solving of the measuring and diagnosing problems is developed in the work. The approach is proposed, the method is developed and the statistics are given to estimate the kernel of the linear random process as electroretinogram model. The obtained results may be used in the applied research of eye diagnosis.
\end{abstract}

Keywords: Electroretinogram, retina, linear random process, mathematical modem, eye, biopotential.

\section{INTRODUCTION}

Despite a lot of methods of investigation, diagnosis, treatment of visual system, accurate disease diagnosing problem remains relevant. Among well-known diagnostic methods a special attention is recently paid to the method of induced potentials, essence of which includes in the diagnosing by analysis of electroretinograms (ERG), each of which is a response to the irritation in the form of instant light impulse.

Computer techniques utilization allowed to systematize present statistical data, but the problem of the early diagnosis and treatment isn't still completely solved. In the eye medical investigation practice the main problem remains in selection of ensuring and appropriate statistical handling of biomedical information which allows registering changes in a human organism.

The analysis of electroretinograms shows that induced potentials of the eye retina contain informative signs of some visual system diseases. As a result there is an urgent task to carry out the statistical handling of electroretinograms with the purpose of diagnostic parameters reveal and diagnosing diseases using them.

For ERG handling methods selection, diagnostic signs selection substantiation it is necessary to determine the ERG model first of all. It is one of the most important points of the signals investigation, including induced potentials, since the ERG handling methods are developed on the basis of the accepted model. The models are divided into descriptive (when the signals are described by the words); physical (object model); graphic (the object is represented by means of some pictures (atom model, chemical compounds, molecules), schemes (electric, mechanical, acoustic); analytical or mathematical.

The purpose of the paper is to develop electroretinogram model.

\section{GENERAL APPROACH FOR ELECTRORETINOGRAM MODEL CONSTRUCTION}

As is well-known living organism structure has a distinct hierarchic character, from the molecular level to the complicated organism systems. For a long time biological objects investigation was carried out on the same level, for instance, molecular, cellular, etc., and the connections between the levels were not taken into consideration. However the recent research reveals that living organisms, especially human organs, are such complicated compounds of dynamically interactive components, that the study of such organisms cannot be effective if it's components are regarded independently of one another. In other words, only examination of all the components as a single unity can give essential information concerning biological system. To carry out such research the necessity of system approach utilization emerges. 
The system means the totality of concerned, interactive elements, which are regarded as a single unity and fulfill some function. Thus, retina may be regarded as the totality of concerned, interactive neurons, the function of which is transformation of the light signal-stimulus to signal-reaction of ganglionic cells, which are concerned with brains.

The signals-stimulus taken to the system, for example visual system retina, are called input signals. Signals which go out of the system or are fixed in some area, are called output. Considering the input-output interaction from the point of view of cause-effect relations and representing such interaction as transformation of the inputs $x_{i}(t)$ to reaction $y_{i}(t)$, such a reaction could be regarded as the function of stimulus:

$$
y_{i}(t)=F\left(x_{1}(t), \ldots, x_{n}(t)\right), \quad i=\overline{1, k} .
$$

Such general approach to the signal model making as "input-output" can be used in electroretinogram model construction. At the same time it is necessary to make some assumptions which meet the physics of the phenomenon.

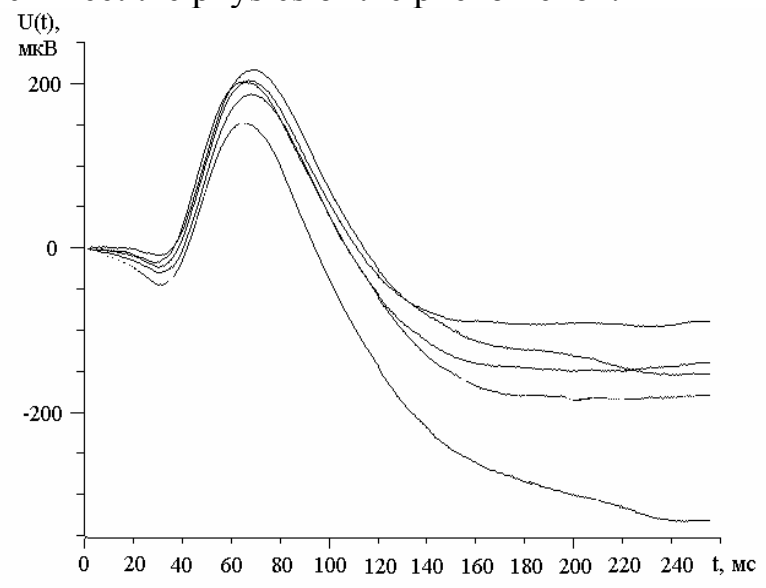

Fig. 1 - General view of the typical electroretinogram

Electroretinogram is an output signal of the visual system, on the entry of which light signals are given. Former ERG analysis reveals that being received in different periods of time they differ one from another, i.e. dispersion is observed. (fig. 1). ERG formation mechanism allows to consider visual system as a linear one. Therefore ERG model will be substantiated as a stochastic process, in the general case - as a stochastic field.

\section{ELECTRORETINOGRAM MODEL MAKING AS A LINEAR STOCHASTIC PROCESS}

Let's assume that the linear system is affected by random impulses, which emerge in casual periods of time. If $\varphi(\tau, t)$-is the impulse response of a vision system, i.e., the response of a nonstationary system at the moment $t$ to a unit impulse that achieves the input at a time moment $\tau$, then ERG can be described by a stochastic process.

$$
\xi(t)=\sum_{k: \tau_{k}<t} \alpha_{k} \varphi\left(\tau_{k}, t\right),
$$

where $\quad\left\{\ldots \tau_{-1}<\tau_{0}<\tau_{1} \ldots<t\right\} \quad$ are time moments of rise of impulses,

$\alpha_{k}$ - are random variables that characterize the intensity of impulses.

Making ERG mathematical model as a linear stochastic process it is convenient to use the process with independent increments. The process is represented by the consequence of random independent impulses, which emerge in random periods of time ${ }^{\tau}{ }_{j}$

$$
\text { Д3 }\left(\phi_{j}\right)={ }_{3}\left(\phi_{j+1}\right)-3(\phi), j=1,2, \ldots
$$

Process increment $\eta(\tau)$ is considered as the influence of the impulse transition function $\varphi(\tau, t)$ on linear system.

The generalization of the model (2) is linear random process $\{22,23\}$

$$
\xi(t)=\int_{-\infty}^{\infty} \varphi(\tau, t) d \eta(\tau)
$$

Where $\eta(\tau)$ - stochastic process with independent increments, the rise points of which coincide with the moments $\tau_{\mathrm{k}}$ in (2), size of jumps are equal $\alpha_{\mathrm{k}}$.

If we assume that the vision system is invariant in time, i.e., for its impulse is response to the equality $\varphi(\tau, t)=\varphi(t-\tau)$, then the model of ERG is a stationary linear process

$$
\xi(t)=\int_{-\infty}^{\infty} \varphi(t-\tau) d \eta(\tau)
$$

Substantiating ERG model as a linear stochastic process (2) or (4) we didn't take into account the space coordinates of the input and output of the system. In the general case, the impulse response depends on time variables $\tau$ and $t$ as well as on space coordinates. In view of the above reasoning, the model of ERG can be represented in the form of a linear stochastic field 


$$
\begin{gathered}
\xi(t, \bar{r})=\int_{-\infty R_{3}}^{\infty} \int_{-\bar{r}} \varphi(\tau, t, \bar{s}, \bar{r}) d_{\tau} d_{s} \eta(\tau, \bar{s}), \\
r \in Q, t \in T,
\end{gathered}
$$

where $\tau, t$ are time variables considered in models (2) - (5);

$\bar{s}$ is the input point of the vision system in the space $R_{3}$;

$\bar{r}$ is the output point of the vision system, i.e. the point at which ERG is observed;

$\varphi(\tau, t, \bar{s}, \bar{r})$ is the impulse space-time response of the linear system;

$\eta(\tau, \bar{s})$ is a nonhomogeneous field with independent increments with respect to time as well as to space, increments of which characterize energetic properties of the incoming signal.

In the case where the vision system is invariant with respect to time, its model is a linear field stationary in time

$$
\xi(t, \bar{r})=\int_{-\infty R_{3}}^{\infty} \int_{R_{3}} \varphi(t-\tau, \bar{s}, \bar{r}) d_{\tau} d_{s} \eta(\tau, \bar{s}) .
$$

Under the condition that, in (6) or (7), the space coordinates $\bar{s}$ and $\bar{r}$ are fixed, we obtain a special case of such models, precisely, the linear process (5) or, respectively, the linear stationary process (4).

According to [17], in technics gilbert linear random processes class is used, which satisfies the condition $\mathrm{M}|\xi(t)|^{2}<\infty$ with every $t \in(-\infty, \infty)$. That is, random process has terminable energy and the process increments disperssion $\eta(\tau)$ is terminable.

If in (4) ) $\eta(\tau)$ is homogeneous stochastic continuous process with independent increments, the logarithm of one-dimensional characteristic function in the Kolmogorov form of process (4) is defined by the expression

$$
\begin{aligned}
& \ln f_{\xi}(u, t)=\ln \mathbf{M}\left[\exp (\operatorname{iu} \xi(t)]=\operatorname{imu} \int_{-\infty}^{\infty} \varphi(\tau, t) d \tau+\right. \\
& +\int_{-\infty}^{\infty} \int_{-\infty}^{\infty}\left[\exp (\operatorname{iux} \varphi(\tau, t)-1-\operatorname{iux} \varphi(\tau, t)] \frac{d K(x)}{x^{2}} d \tau\right.
\end{aligned}
$$

where $m$ is the mathematical expectation and $K(x)$ is the Poisson spectrum of jumps on the Kolmogorov form which are found in formulas (3-5)

It is easy to show that, in the case where the generating process $\eta(\tau)$ of the linear stochastic process (4) is homogeneous, the mathematical expectation for (4) is given by the formula

$$
\mathbf{M}[\xi(t)]=\chi_{1} \int_{-\infty}^{\infty} \varphi(\tau, t) d \tau
$$

and the correlation function is represented by the expression

$$
R_{\xi}\left(t_{1}, t_{2}\right)=\chi_{2} \int_{-\infty}^{\infty} \varphi\left(\tau, t_{1}\right) \varphi\left(\tau, t_{2}\right) d \tau
$$

where $\chi_{1}$ and $\chi_{2}$-are the corresponding cumulants of the random variable $\eta(1)$.

For the stationary linear stochastic process (5), expressions (6) and (7) take the form

$$
\begin{gathered}
\mathbf{M}[\xi(t)]=\chi_{1} \int_{-\infty}^{\infty} \varphi(x) d x=m=\text { const } \\
R_{\xi}(\tau)=\chi_{2} \int_{-\infty}^{\infty} \varphi(x) \varphi(x+\tau) d x .
\end{gathered}
$$

In models (2), (4), (5), we do not take into account the space coordinates of the input and output of the system. In the general case, the impulse response depends on time variables $\tau$ and $t$ as well as on space coordinates. In view of the above reasoning, the model of ERG can be represented in the form of a linear stochastic field

$$
\begin{gathered}
\xi(t, \bar{r})=\int_{-\infty R_{3}}^{\infty} \int_{\bar{r} \in Q, t \in T,} \varphi(\tau, t, \bar{s}, \bar{r}) d_{\tau} d_{-s} \eta(\tau, \bar{s}), \\
\bar{r},
\end{gathered}
$$

where $\tau, t$ are time variables considered in models (2), (4), (5);

$\bar{s}$ is the input point of the vision system in the space $R_{3}$;

$\bar{r}$ is the output point of the vision system, i.e. the point at which ERG is observed;

$\varphi(\tau, t, \bar{s}, \bar{r})$ is the impulse space-time response of the linear system;

$\eta(\tau, \bar{s})$ is a nonhomogeneous field with independent increments with respect to time as well as to space, increments of which characterize energetic properties of the incoming signal.

In the case where the vision system is invariant with respect to time, its model is a linear field stationary in time 


$$
\xi(t, \bar{r})=\int_{-\infty R_{3}}^{\infty} \int_{R} \varphi(t-\tau, \bar{s}, \bar{r}) d_{\tau} d_{s} \eta(\tau, \bar{s}) .
$$

It is important that the characteristic function of the linear stochastic process (4) is written in the explicit form. If the generating process $\eta(\tau)$ is homogeneous, then, by virtue of [1], the logarithm of one-dimensional characteristic function in the Kolmogorov form of process (3) is defined by the expression

$$
\begin{aligned}
& \ln f_{\xi}(u, t)=\ln \mathbf{M}[\exp (i u \xi(t)]= \\
& =\operatorname{imu} \int_{-\infty}^{\infty} \varphi(\tau, t) d \tau+ \\
& +\int_{-\infty}^{\infty} \int_{-\infty}^{\infty}\left[\begin{array}{l}
\exp (\operatorname{iux} \varphi(\tau, t)-1- \\
-\operatorname{iux} \varphi(\tau, t)
\end{array}\right] \frac{d K(x)}{x^{2}} d \tau,
\end{aligned}
$$

where $m$ is the mathematical expectation and $K(x)$ is the Poisson spectrum of jumps on the Kolmogorov form.

The method of characteristic functions is important in practical sense for the solution of many applied problems, furthermore, the following statements are important [4,5]:

1) there exists the one-to-one correspondence between distribution functions and characteristic functions;

2) when characteristic functions are used, in view of their continuity and boundedness, the classical methods of the functional analysis are applied that makes it possible to analyze various types of convergence of series of characteristic functions instead of distribution functions;

3) with the use of characteristic functions the principal results of the theory of probability related to the proving central limit theorem for the sum of independent random variables are obtained;

4 ) in the case of the existence of the first $n$ moments, the characteristic function has continuous derivatives till $n$ order inclusively that makes it possible to simplify considerably the process of calculation of moments (cumulants) of $n$ the order of a random variable with the use of the characteristic function.

As was indicated in [3], the general view of the characteristic function (15), enables one to analyze completely the responses of linear systems: to find cumulants, distribution function or distribution function of the response, to investigate the distribution of jumps of incoming and outgoing realizations of the system, to establish the relationship of incoming and outgoing characteristics of linear sections.

For the linear random process (5) the expressions for the average of distribution and correlation function are the following:

$$
\begin{array}{r}
M[o(t)]=\kappa_{1} \int_{-\infty}^{\infty}(\phi t) d \phi, \\
R_{o}\left(t_{1}, t_{2}\right)=\kappa_{2} \int_{-\infty}^{\infty}\left(\phi t_{1}\right)\left(\phi t_{2}\right) d \phi,
\end{array}
$$

where $\kappa_{1 \mathrm{i}} \kappa_{2}$ - the corresponding cumulants of the random variable $\eta(1)$.

In case of stationary linear random process (5) the expressions (12) and (13) are represented as

$$
\begin{gathered}
M[o(t)]=\kappa_{1} \int_{-\infty}^{\infty}(x) d x=\text { const } \\
R_{o}(\phi)=\kappa_{2} \int_{-\infty}^{\infty}(x)(x+\phi) d x .
\end{gathered}
$$

Model of ERG in the form of linear stochastic process with discrete time. By virtue of the fact that an information measuring system (IMS) includes arrangements that realize the digital signal processing (digitalization with respect to time and quantification with respect to the level of real continuous signals), it is important to construct a model of ERG with discrete time.

According to [3-5] a linear stochastic process with discrete time is a process

$$
\xi_{t}=\sum_{\tau=-\infty}^{\infty} \varphi_{\tau, t} \varsigma_{\tau}, t \in(\overline{-\infty, \infty})
$$

formed by a linear system with discrete impulse response $\varphi_{\tau, t}, \quad \sum_{\tau=-\infty}^{\infty}\left|\varphi_{\tau, t}\right|^{2}<\infty$ for every fixed $t$ with the use of input white noise $\varsigma_{\tau}, \tau \in(-\infty, \infty)$. The process $\varsigma_{\tau}$ is called a generating process. The nonrandom function $\varphi_{\tau, t}$ is called the kernel of representation (2.16).

The mathematical expectation and correlation function for LSP with discrete time is as follows:

$$
\mathrm{M} \xi_{t}=\kappa_{1} \sum_{\tau=-\infty}^{\infty} \varphi_{\tau, t}
$$




$$
R_{t_{1}, t_{2}}=\kappa_{2} \sum_{\tau=-\infty}^{\infty} \varphi_{\tau, t_{1}} \varphi_{\tau, t_{2}}
$$

where $\chi_{1}=\mathbf{M} \varsigma_{\tau}, \chi_{2}=\mathbf{D} \varsigma_{\tau}$.

In the case where LSP is stationary, i.e., $\xi_{t}=\sum_{\tau=-\infty}^{\infty} \varphi_{\tau-t} \varsigma_{\tau},(17)$ and (18), we have

$$
\begin{gathered}
\mathrm{M} \xi_{t}=\kappa_{1} \sum_{x=-\infty}^{\infty} \varphi_{x}=\text { const }, \\
R_{\tau}=\kappa_{2} \sum_{x=-\infty}^{\infty} \varphi_{x} \varphi_{x+\tau}
\end{gathered}
$$

\section{Conclusions}

In the present paper the electroretinogram model is developed in the form of a linear stochastic process taking into account the mechanism of eye retina formation. It is important that the developed model suits for solving measuring and diagnosing tasks.

\section{REFERENCES}

[1] Shpak A.A. Investigation of Visual GammaInduced Potentials in Ophthalmology and Ophthalmosurgery. - Moscow: MSTC "Microsurgery of eye", 1993. - $191 \mathrm{p}$.

[2] Shpak A.A. Evaluation of Visual GammaInduced Potentials in Dependence of Visual Stimulation Character // Transactions of Ophthalmology. - 1991. - Vol.107, No.3. -Pp. 32-39.

[3] Marchenko B. G. Method of Stochastic Integral Representations and its Applications in Radio Ingineering, Naukova Dumka, Kiev (1973).

[4] Marchenko B. G. and Shcherbak L. N. Linear Stochastic Processes and Their Application, Naukova Dumka, Kiev (1975).

[5] Marchenko B. G. and Myslovich M. V. Vibrodiagnosis of Bearing Knots of Electric Machines, Naukova Dumka, Kiev (1992).

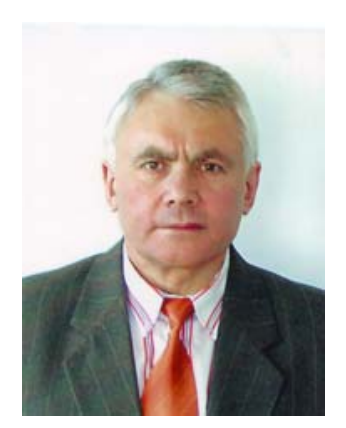

Mykola Pryjmak, Dr. of Technical Sciences, Professor, Department of Computer Sciences, Ivan Pulyuj Ternopil State Technical University.

His research interests are: fundamental issues in periodical stochastic processes and noices with application in power industry and biomedicine; theory of periodical functions and processes, variable period processes and periodical processes with functional dependence.

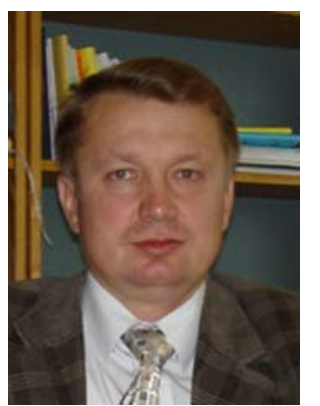

Oleksandr

Matsyuk, Candidate of Technical Sciences, Associate Professor, Chair of Department of Computer Sciences, Ivan Pulyuj Ternopil State Technical University.

His research interests are: statistical theory of decision making; processing

biomedical signals; power saving technology.

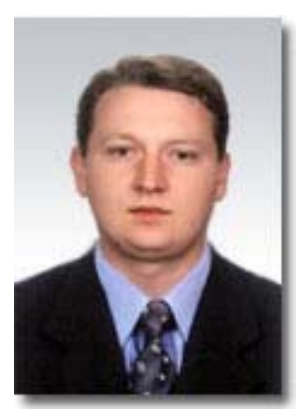

Serhij Martsenko, graduate student in "Mathematical simulation and computing", Department of Computer Sciences, Ivan Pulyuj Ternopil State Technical University.

Serhij Dzyubin, graduate student in "Mathematical simulation and computing", Department of Computer Sciences, Ivan Pulyuj Ternopil State Technical University. 